\title{
Analysis of the stress state of the ribbed-ring metal dome under different methods of installation
}

\author{
Evgeny Lebed*
}

Moscow State University of Civil Engineering, Yaroslavskoe shosse, 26, Moscow, 129337, Russia

\begin{abstract}
A computer model of the frame of a ribbed-ring metal dome of steel I-beams with a span of $30,3 \mathrm{~m}$ and a height of $7,5 \mathrm{~m}$ with rigid connections at the joints have been developed. Based on the design scheme, auxiliary models of a partially complete dome frameworks were designed to study alternative installation methods, which differ from each other in the location scheme of temporary supports with hinge joints of bar elements over them. The installation computer models utilized the three schemes of temporary supports: central support, central support and 1 circular row of intermediate supports, central support and 3 circular rows of intermediate supports. Computer calculations were performed for the design model of the dome framework and for all installation models to evaluate the impact of the self-weight of the dome on the stress state of the elements of its framework. As a result of the research, graphs and diagrams were created, which reflect the level of installation stresses in the meridional ribs in comparison with their values in the design scheme. The necessity to perform calculations of the dome frameworks during the design according to the installation conditions is noted.
\end{abstract}

\section{Introduction}

Domes are used as roofs of buildings that are round in plan, or in combination with other span structures. They are widely used not only because of their expressive geometric shape, but also because of their high reliability. The circular symmetry gives them increased rigidity compared to other spatial structural systems [1]. This, combined with the costeffectiveness of metal construction, allows them to occupy a leading position in the category of large-span spatial roofs.

The frames of metal domes cannot be prefabricated and brought to the construction site as a whole structure. They acquire their design form, which is realized in their external appearance, gradually, over a long time. At first, only individual structures appear, then these structures are combined into fragments of the dome frameworks, those fragments are enlarged (or combined with each other) to form a single dome framework. Dome frameworks are assembled from a large number of metal structures, which are also differently oriented in space. The complexity of the structural system of domes and the

\footnotetext{
*Corresponding author: evglebed@mail.ru
} 
method of their installation, in many respects, depends on the magnitude of their dimensions and the geometric scheme of their frames [2].

Real structures in metal domes have their own weight, and their frameworks are formed gradually during installation. Because of this, during the installation process, individual elements and structures are often installed on temporary supports. The installation diagram of the structures or parts of the dome frameworks in this case differs significantly from the design scheme. Therefore, during the construction of domes, internal forces arise in the structural elements of their frames, which differ in nature and magnitude from those that arise when the frames work according to design schemes.

The erection of the frames of large-span metal domes can be carried out in various ways [3]: with a temporary central support, with several temporary supports, with scaffolding, lifting after assembly on the ground, mounted installation. A temporary central support is installed under the top of the dome, usually the upper ring of ribbed-ring domes is arranged on it. If several temporary supports are used, then in addition to the central one, a number of several supports are installed at an equal distance from it (along the circumference). There may be several such rows, and the axes of these rows resemble concentric circles of different diameters. With any method of erection dome frameworks are assembled both from individual structures and from fragments of frameworks, of varying degrees of enlargement $[4,5]$.

The object of the study was the framework of the ribbed-ring dome, the geometric shape of which is shown in Fig. 1. The dome is spherical with a radius of curvature of $19 \mathrm{~m}$, a span of $30,3 \mathrm{~m}$ and a height of $7,5 \mathrm{~m}$. The frame of the dome consists of 24 meridional ribs and has 5 rings, the diameter of the upper ring is $5,4 \mathrm{~m}$. The length of a single element of the meridional rib between the rings is $3,7 \mathrm{~m}$. The lengths of individual elements of the rings vary from $0,7 \mathrm{~m}$ at the top of the dome to $3,95 \mathrm{~m}$ at the reference contour.

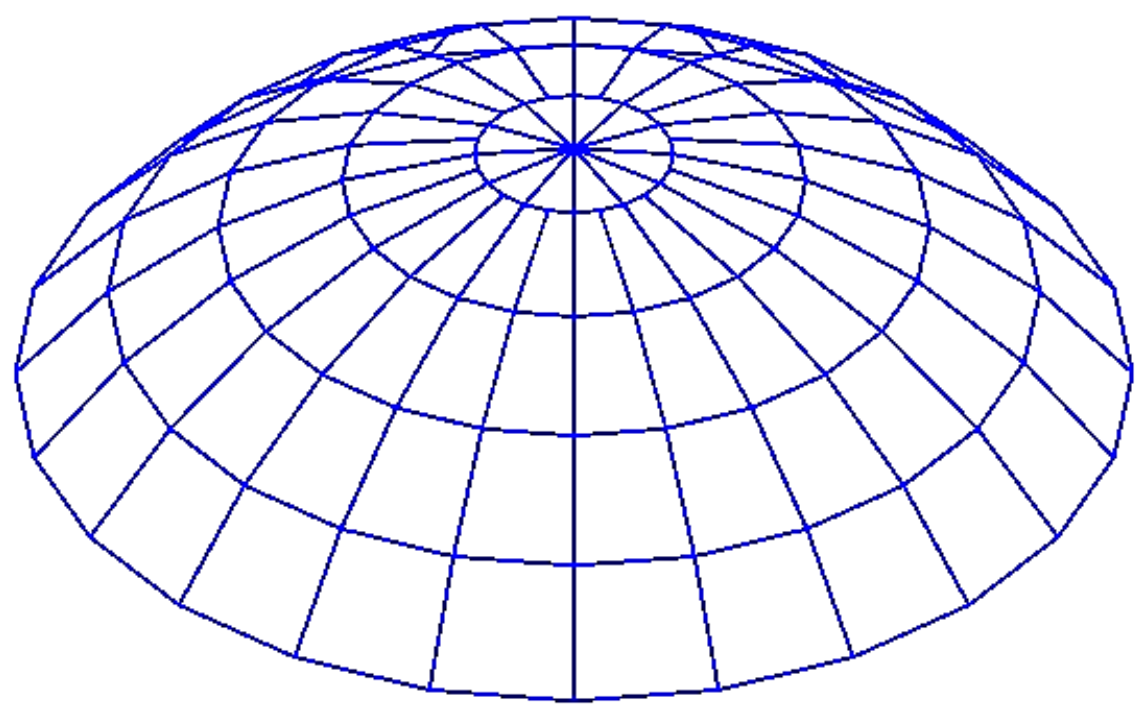

Fig. 1. Geometric diagram of the dome under study. 
The study of the stress state of the frame during the installation of the ribbed-ring dome was carried out in various ways with the SCAD software system [6] on specially developed computer models of spatial rod systems [7]. The dome framework consists of steel I-beams: meridional ribs of I 23Ш1, the upper ring of I 26Ш1, support ring of I $23 \amalg 1$, intermediate rings of I $20 \amalg 1$. The dome framework of the design scheme is supported along its contour on short permanent columns of I 35W1. All temporary support columns are made of $180 \times 5 \mathrm{~mm}$ pipes.

\section{Methodology}

The study of the stress state of framed structures of a dome type with the use of computer models is typical for different scientists. Usually the stress state of dome frameworks was analyzed when its geometric parameters changed, when the geometric shapes of the frameworks differed, when the height-to-diameter ratio of the dome was different [8], when the spans of the dome frameworks changed [9]. Previously the author carried out a comparative study of the ribbed-ring and annular- lattice domes with different installation methods [10].

The dome framework is a spatial structure with rigid connections between all the elements, freely installed on permanent supports under the lower ring. Therefore, in this study, the design model of a dome framework is a framed structure with rigid joints supported by short columns through hinge joints. For computer installation models of a partially complete framework, temporary supports were used: a central support, a central and 1 circular row of intermediate supports, a central support and 3 circular rows of intermediate supports (Fig. 2).

During the installation process, the individual parts of the frameworks on the supports are not yet rigidly connected to each other; nevertheless, they are installed on temporary supports without displacement. Therefore, the dome frameworks on an installation diagram are bar systems with hinge connections between the installed elements of the meridional ribs to the lower and upper rings, as well as to the intermediate rings of the dome (Fig. 3). The connections with the ribs of intermediate rings and the connections of all the temporary columns with the joints of the dome framework are also hinge. Each of these models is equivalent to the corresponding states of the dome being installed with its specific structural design.

Different structural designs lead to different results of the static operation of framed constructions in dependence on the self weight of installed the meridional ribs, their fragments or individual elements. Therefore, the outlines of deformed dome structures with meridional ribs will differ in the wiring diagrams and different in the design scheme (Fig. 4). The diagrams of bending moments in the meridional ribs also become different; they differ both from each other and from the diagrams of the design scheme (Fig. 5). The arrangement of rigid joints between the elements of the meridional ribs and the installation of the dome ring elements in the joints with the formation of closed contours fix the deformations of the ribs and, thereby, contributes to the preservation of part of the installation stresses caused by bending. 

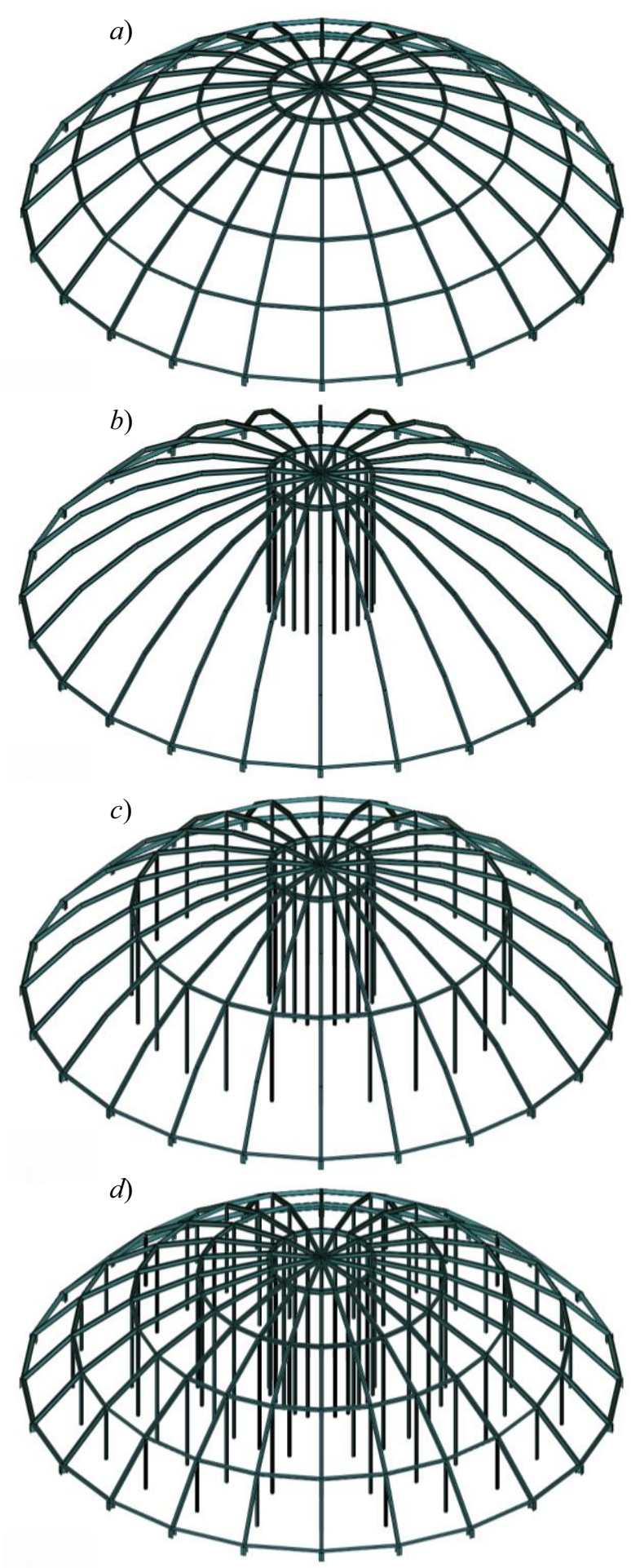

Fig. 2. The models of the dome framework under study

$a$ - design scheme; $b$ - central support; $c$ - central support and 1 circular row of supports; $d-$ central support and 3 circular rows of supports. 

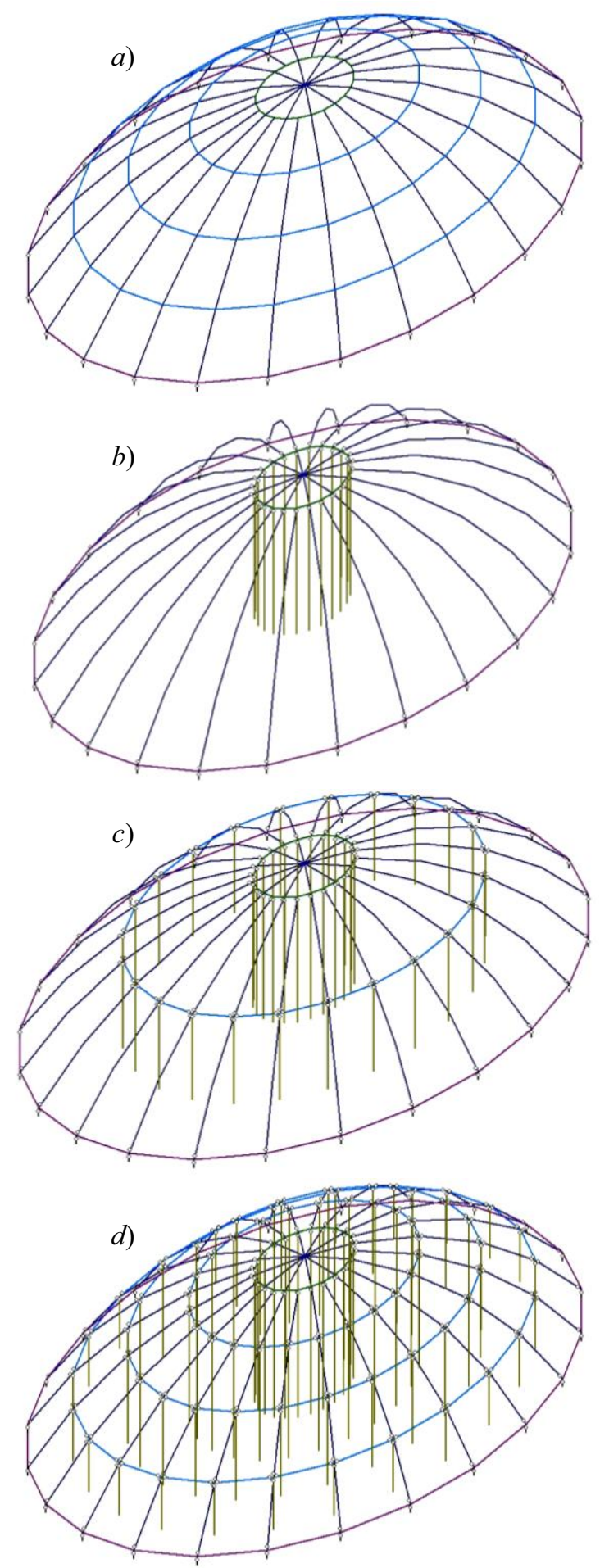

Fig. 3. Design scheme of a dome framework during installation $a$ - design scheme; $b$ - central support; $c$ - central support and 1 circular row of supports; $d-$ central support and 3 circular rows of supports. 

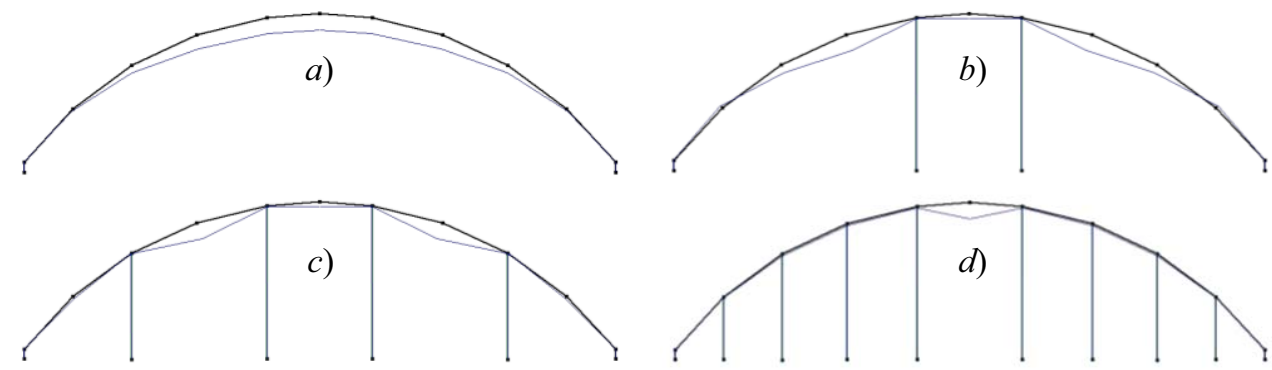

Fig. 4. Deformations of the meridional ribs of different design schemes of a dome framework $a$ - design scheme; $b$ - central support; $c$ - central support and 1 circular row of supports; $d-$ central support and 3 circular rows of supports.
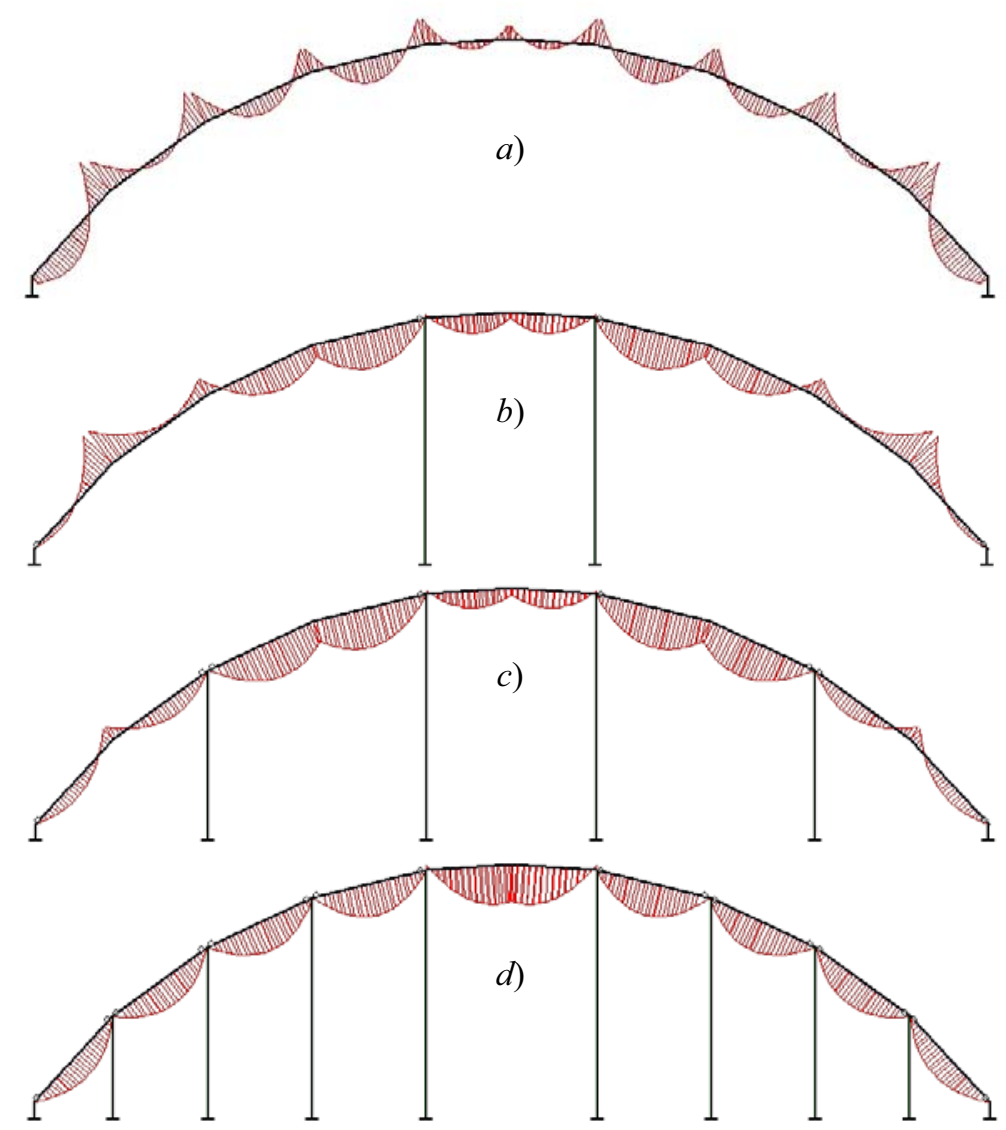

Fig. 5. Diagrams of bending moments $M_{x}$ in the meridional ribs of different design schemes of a dome framework

$a$ - design scheme; $b$ - central support; $c$ - central support and 1 circular row of supports; $d-$ central support and 3 circular rows of supports. 


\section{Example}

The resulting internal forces $N_{i}$ and $M_{i}$ in the elements of the meridional ribs of each of the structural designs of a dome framework are characterized by a range of values and different arithmetical signs: $M_{i}$-the moment from above; $M_{i}$ the moment from below. However, the meridional ribs of the dome are always in a state of compression with a bend, regardless of the moment's sign $M_{i}$. Therefore, the comparison of moments $M_{i}$ in different designs is justified. Fig. 6 shows diagrams of moments in different computer models $M_{j, i}$ of a framework $j$ in different tiers of ribs $i$ in comparison with the maximum moment of the design scheme $M_{\Pi, \max }$ (the first tier) in terms of the ratio values $M_{j, i} / M_{\Pi, \max }$. The diagrams show that the bending moments of installations can exceed the maximum moment of the design scheme with listed above installation methods by 1,$67 ; 1,62 ; 1,49$ times, respectively.

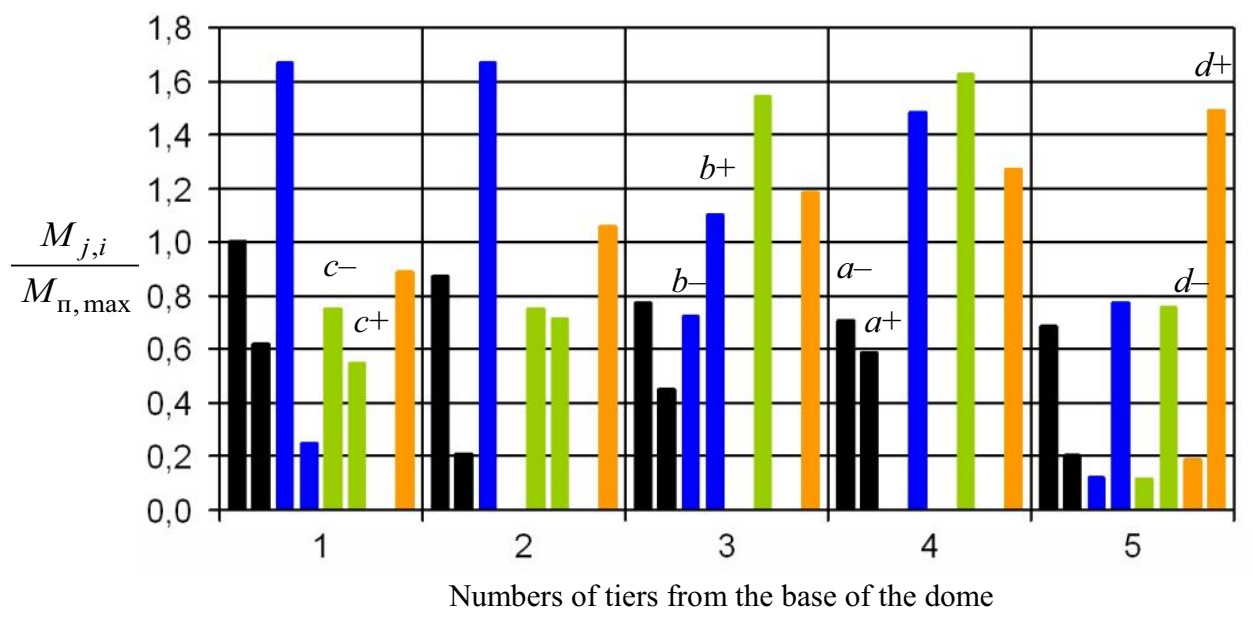

Fig. 6. The ratio $M_{j, i} / M_{\Pi, \max }$ in the elements of the ribs of different design schemes of a dome framework

$a$ - design scheme; $b$ - central support; $c$ - central support and 1 circular row of supports;

$d$-central support and 3 circular rows of supports; "-" moment from above; "+" moment from below.

The normal stresses in the elements of the meridional ribs are composed of the sum of the stresses from compression and the stresses from bending, i.e.

$$
\sigma_{i}=\sigma_{N i} \pm \sigma_{M i}
$$

The self weight of the elements of the dome framework is a vertical load, therefore $M_{y, i}=0$. Therefore, to calculate the normal stresses in the cross-sections of the elements of the meridional ribs, we can use the formula

$$
\sigma_{i}=\frac{N_{i}}{\mathrm{~A}_{i}} \pm \frac{M_{x, i}}{\mathrm{~W}_{x, i}}
$$


The stresses in the elements of the meridional ribs of the dome framework design scheme $\sigma_{\Pi i}=\sigma_{N \Pi i} \pm \sigma_{M \Pi i}$ depend more on the longitudinal forces $N_{i}$ than on the bending $M_{i}$. So, for $M_{i}-$ the ratio $\sigma_{N i} / \sigma_{\Pi i}$ is in the range from 0,60 to 0,67 , and for $M_{i}+$ the ratio $\sigma_{N i} / \sigma_{\Pi i}$ is in the range from 0,66 to 0,89 (Fig. 7).

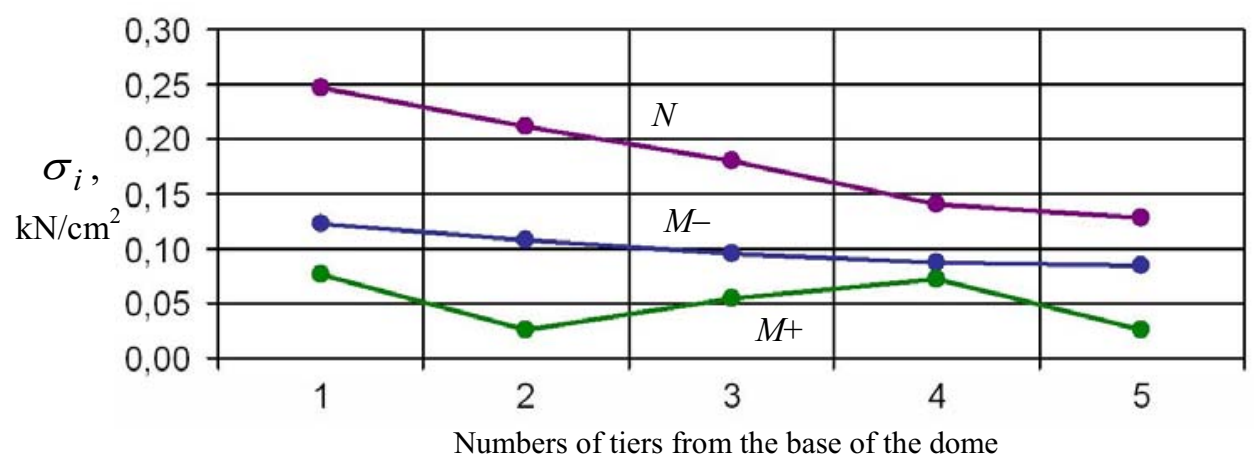

Fig. 7. The normal stresses in the cross-sections of the meridional ribs of design scheme $\sigma_{\Pi i}$ from the longitudinal force $N$ and the bending moments $M-, M+$.

If a share of bending stresses in the meridional ribs of the dome framework design scheme $\sigma_{M_{\Pi i}}$ is replaced by similar share of bending stresses in the installation $\sigma_{M_{м} i}$, then we get the initial stresses $\sigma_{\mathrm{H} i}=\sigma_{N_{\Pi i}} \pm \sigma_{M \mathrm{M} i}$ that the meridional ribs will experience at the end of the installation. Fig. 8 shows diagrams of designed $\sigma_{\Pi i}$ and initial $\sigma_{\mathrm{H} i}$ stresses in the meridional ribs of the dome framework. The diagrams show that the initial stresses $\sigma_{\mathrm{H} i}$ in the dome ribs differ from the designed ones and significantly exceed them on certain tiers. However, it should be noted that the values of the initial stresses on the upper tiers do not exceed the maximum design stresses $\sigma_{\Pi \max }$.

To quantify these differences, diagrams of the ratio of initial stresses to design stresses were constructed $\sigma_{\mathrm{H} i} / \sigma_{\Pi i}$ (Fig. 9). The diagrams show that when installed with a central support for $M_{i}-$, the initial stresses $\sigma_{\mathrm{H} i}$ can exceed those designed $\sigma_{\Pi i}$ by 1,31 times and exceed the maximum $\sigma_{\Pi \max }$ ones by 1,22 times, and for $M_{i}+$ stresses $\sigma_{\mathrm{H} i}$ can exceed the designed values $\sigma_{\Pi i}$ by 1,52 times in the 4 th tier. When installed with a central support and 1 circular row of supports for $M_{i}+$ the initial stresses $\sigma_{\mathrm{H} i}$ can exceed those designed $\sigma_{\Pi i}$ by 1,60 times in the 4th tier. When installed with a central support and 3 circular rows of supports for $M_{i}+$ the initial stresses $\sigma_{\mathrm{H} i}$ can exceed those designed $\sigma_{\Pi i}$ by 1,44 times in the 2 nd tier and 2,04 times in the 5 th tier. 


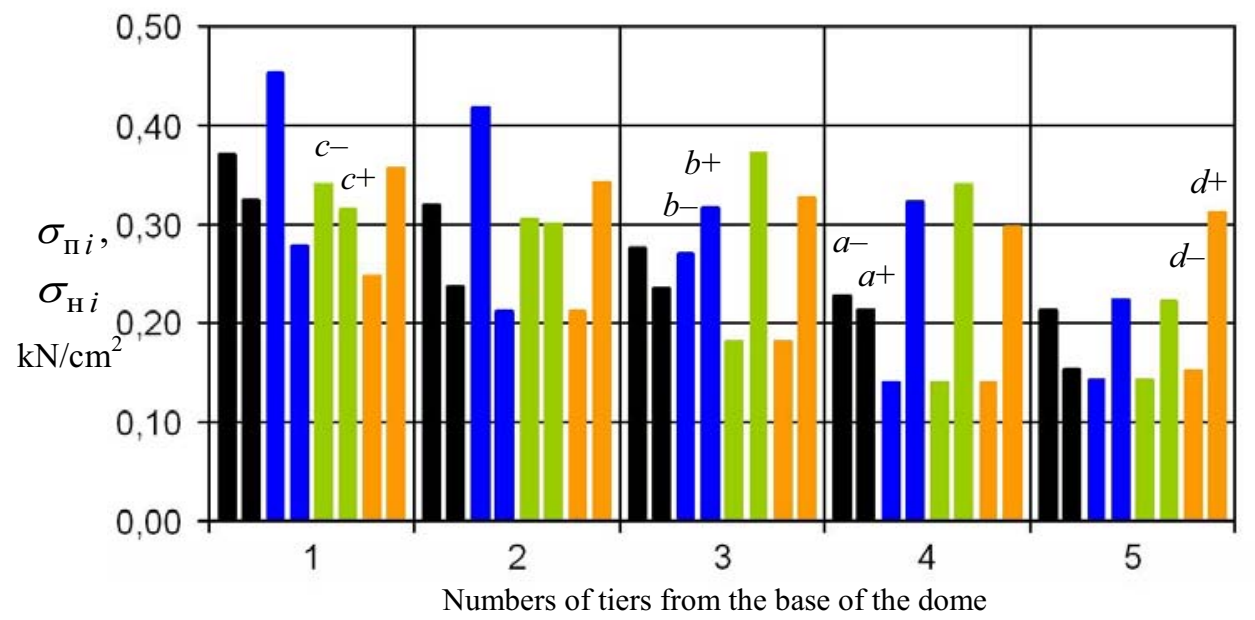

Fig. 8. Designed $\sigma_{\Pi i}$ and initial $\sigma_{\mathrm{H} i}$ stresses in the meridional ribs of the dome

$a$ - design scheme; $b$ - central support; $c$ - central support and 1 circular row of supports; $d$-central support and 3 circular rows of supports; "-" moment from above; "+" moment from below.

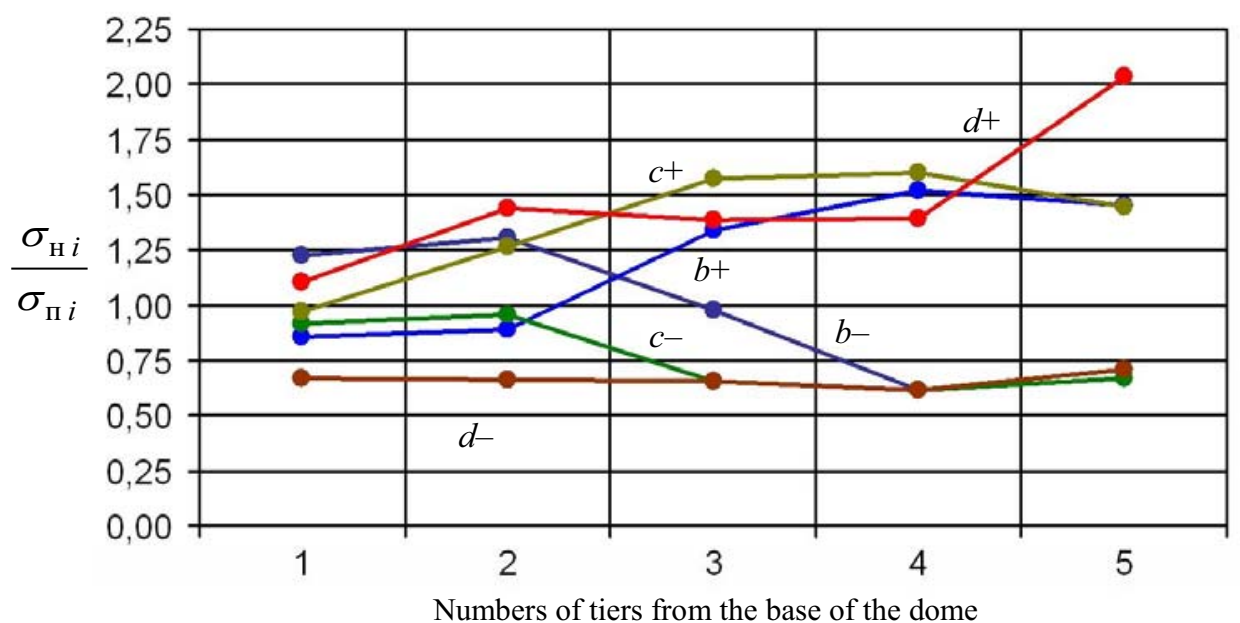

Fig. 9. The ratio of the initial stresses to the design stresses $\sigma_{\mathrm{H} i} / \sigma_{\Pi i}$ in the dome ribs $b$ - central support; $c$ - central support and 1 circular row of supports; $d$-central support and 3 circular rows of supports; "-" moment from above; "+" moment from below.

\section{Conclusions}

During the installation of a ribbed-ring metal dome, stresses occur in the meridional ribs whenever what the installation method is. The reason for the installation stresses is the fact that during the installation process, the structural design of the elements of the dome frameworks differ from the design scheme. The level of the values of the installation 
stresses, their nature and distribution over the tiers depend on the installation methods. However, in general, the lower the number of temporary supports used in the installation of dome frameworks, the higher the absolute values of the stress.

The values of the installation stresses in the meridional ribs for different installation methods are comparable to the stresses from the self weight of the framework in the design scheme. For different tiers of the dome framework, installation stresses can lead to initial forces that are significantly higher than those determined in the design scheme. For example, when installing with a central support, the initial stresses in the ribs may exceed the maximum design stresses by $22 \%$. At the same time, in relation to the stresses in the ribs on different tiers, the initial stresses may exceed the design ones by more than $50 \%$.

Despite the relatively low level of stress in the meridional ribs of the design schemes of dome frames from the load of the self weight, it is necessary to calculate the frames of metal domes for all types of installation schemes. For absolute confidence in ensuring reliability, the results of calculations should be taken into account when evaluating the performance of the dome frames for operational loads.

\section{References}

1. Krivoshapko S.N. Metal ribbed-and-circular and lattice shells from the XIX ${ }^{\text {th }}$ until the first half of the $\mathrm{XX}^{\text {th }}$ centurie / Structural Mechanics of Engineering Constructions and Buildings (2014) No 6 Pp 4-15.

2. Kuznetsov V.V., Metal Structures. Vol. 2. Steel structures of buildings and constructions. Reference book the designer. Moscow: ASV publ. (1998) 512 p.

3. Gofshteyn G.E., Kim V.G., Nishchev V.N., Sokolova A.D. Installation of Metal and Reinforced Concrete Structures. Moscow: Stroyizdat publ. (2004) 528 p.

4. Lebed E.V. Behavior of the Frames of Large-span Metal Domes in the Process of their Installation / Structural Mechanics of Engineering Constructions and Buildings (2018) 14(6) Pp 481- 494.

5. Youichi M., Terumasa F., Yoshihiko K., Takashi U. Erection Methods for Space Structures / Evolution and Trends in Design, Analysis and Construction of Shell and Spatial Structures / Proceedings of the International Association for Shell and Spatial Structures (IASS) Symposium. Valencia, Spain (2009) Pp 1951-1962.

6. Karpilovskiy V.S., Kriksunov E.Z., Malyarenko A.A., $\quad$ Perel'muter A.V., Perel'muter M.A. SCAD Office. Computer system SCAD: - M.: ASV Publ. (2004) $592 \mathrm{p}$.

7. Gorodetskiy A.S., Evzerov I.D. Computer models of structures - Kiev: "Fakt" Publ. (2005) $344 \mathrm{p}$.

8. Chacko P., Dipu V.S., Manju P.M. Finite Element Analysis of Ribbed Dome // International Journal of Engineering Research and Applications (IJERA) Kerala, India, (2014) ISSN: 2248-9622. Pp 25-32.

9. Merilmol Eldhose, Rajesh A.K., Ramadass S. Finite Element Analysis and Parametric Study of Schwedler Dome Using ABAQUS Software / International Journal of Engineering Trends and Technology (IJETT) - Kerala, India (2015) Vol. 28, No 7. Pp 333-338.

10. Lebed E.V. Computer Analysis of the Behavior of Large-span metal Domes with differenr methods of Installation / Structural Mechanics of Engineering Constructions and Buildings (2018) 14(4) Pp 261-272. 